\title{
Experimental Study of the convective heat transfer coefficient in electronic cooling
}

\author{
by M. REBAY ${ }^{1,{ }^{*}}$, R. BEN MAAD ${ }^{2}$, S. KAKAÇ ${ }^{3}$ and J. PADET ${ }^{1}$ \\ ${ }^{1}$ UTAP - Lab. de Thermomécanique (EA 3082) Faculté des Sciences, 51687 Reims, \\ France \\ ${ }^{2}$ LETTM, Faculté des Sciences de Tunis, 2092 Manar II, Tunisia \\ 3 Turkish Academy of Sciences, Department of Mechanical Engineering, University of \\ Miami, Coral Gables FL 33124, USA \\ (mourad.rebay@univ-reims.fr)
}

\section{Abstract}

As a contribution to the development of the thermal management of the cooling of electronic, we present here the cartographies of the thermal field during the real functioning conditions of a main printed wiring board of a PC. We present also the method of evaluation of the convective heat transfer coefficient between one component (i.e. the main microprocessor) and an air flow in the direction parallel to the printed board. This method consists of analysing the transient temperature of the component, assuming one dimensional conduction in the wall, after a sudden supply of luminous energy by two lamps. Results for three cooling situations are presented.

\section{Introduction}

As the electronic industry tends towards microprocessors and integrated circuits to be faster and smaller, the heat flux that must be extracted becomes higher. Thus, the electronic cooling becomes more than ever a critical task to develop faster and miniaturized electronic components. To cope with the increase of cooling requirements, several theoretical and experimental studies have been carried out to understand convective heat transfer in micro sized systems. Because of the high heat capacity and thermal conductivity of liquids, many studies have been focused on single - phase flow of liquid or on phase change from liquid state to vapour state. Due to the advantages of the maintenance facility and of the relatively low cost in cooling with airflow, considerable attention has been given to thermal control and management techniques with gaseous flow. Various studies in both liquid and gaseous flows in microscale heat transfer are summarized in [1].

By considering that the air-cooling is still the most useful technique in power electronic used in personal computer which commercialised nowadays, and probably in the next decennia, it is very important to develop strategies for finding the optimal cooling systems with air and design configurations. The increase of the heat transfer quantities extracted from the integrated circuits and the microprocessors can be obtained by several ways: optimisation of the location and the direction of the cooling fans, suitable choice of the geometry and the material of the heat sink and of the heat spreader, typical arrangement of the air-cooled modules on the printed wiring board $[2,3]$.

As contribution to the development of the cooling techniques, we are interested in investigating the situations where the control of the structure of the flows in the box which contains the electronic cards would create augmentation of the heat flux extracted by the air flow. The objective is to give minimum changes on the commercially available electronic board. In order to improve the cooling efficiency by the control of the flow structure, it would be interesting to create, with the need of flow 
disturbers, of some zones with artificial turbulence and acceleration of the air flow. One way to obtain this is the implementation on the electronic cards or in the PC box of deflectors, which allow guiding the flow to the regions where a supplementary cooling is necessary (i.e. around the microprocessor package). The ideal situation is that deflectors create turbulence of the flow with minimum increase in the pressure drop. To study the efficiency of such management it is important, as a first step, to develop a reference test. This test should give the reference database for the comparison of the results of the management solutions. For these reasons, we present here, the measurement results of the thermal field on a main electronic printed circuit board used in a personal computer during its real functioning conditions. In addition, we give a method for the measurement of the heat transfer coefficient between the microprocessor package and the air flow supplied by the fan.

\section{Experimental set-up}

(9)

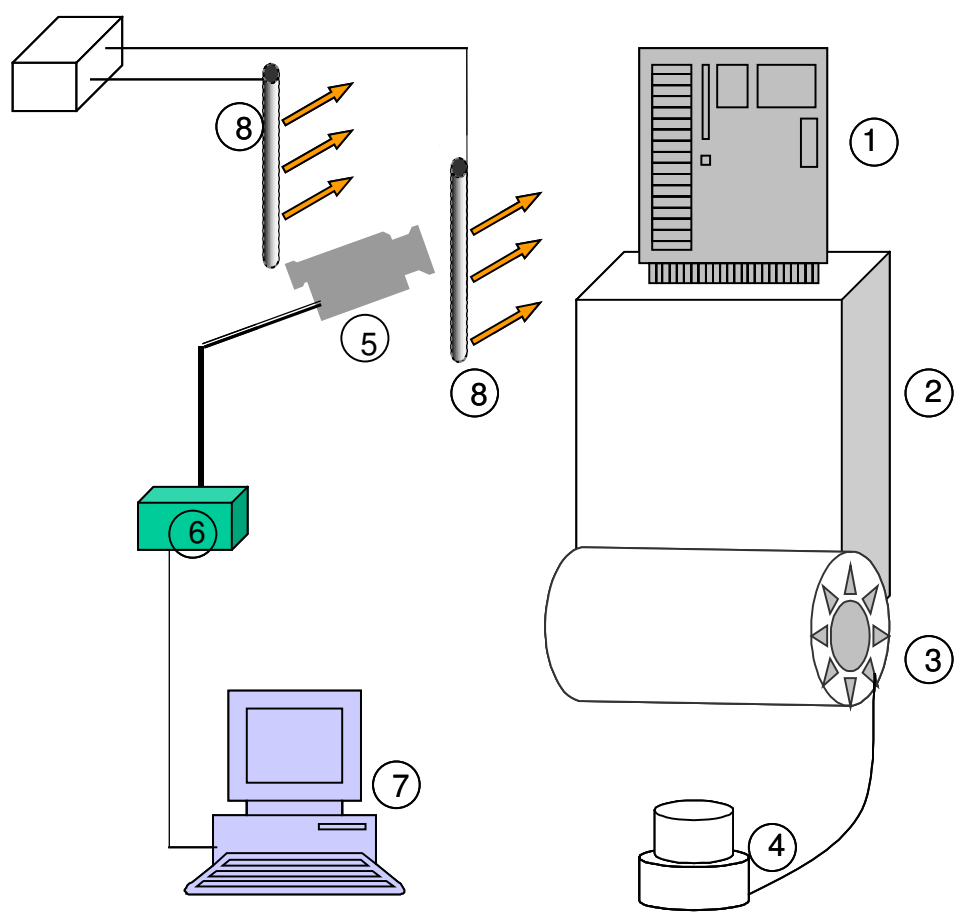

Fig. 1. Experimental set-up

We realised an experimental set-up schematised on Figure 1 for the measurement, by an infrared camera, of both temporal and spatial evolution of the temperature on the front surface of a main printed wiring board. Firstly, the personal computer was dismantled and all their components (drivers, electronic cards, alimentation box...) are given out of its box. The main board (1) was maintained vertically at the outlet of a rectangular channel with $300 \times 250$ cross flow section (2). The airflow, generated by a double aspiration fan (3), was directed via a flow calming section in the channel with $700 \mathrm{~mm}$ in straight length. This channel allows having the 


\section{http://dx.doi.org/10.21611/qirt.2006.085}

airflow to be parallel to the main board and covering the entire of its width. An AC converter voltage (4) controls the fan. The flow velocity associated with each voltage used in the tests was preliminary measured by a propeller anemometer on the outlet section of the channel. Several flow calming sections were also previously tested in order to give maximum uniformity of the flow velocity at the outlet of the channel.

The thermal cartographies were obtained by a short-wave $(3.4-5 \mu \mathrm{m})$ infrared camera (5) with Stirling cycle cooling (FLIR, SC1000). The camera has a matrix with 256x256 active PtSi/CMOS elements. For recording the infrared video, the camera was connected to the digital interface box (6). A cable lies the interface box to a break out box, from which a second cable is connected to the PCMCIA card interface mounted on a station (7). A software allows recording by the station of the infrared images with $50 \mathrm{~Hz}$ sample rate. The details of the temperature field around one selected component may be given by a $100 \mu \mathrm{m}$ macro-objective.

In usual functioning of personal computers, the integrated circuit don't work with the same clock frequency, they depend on the needs of executed programs. So, the quantity of heat that must be dissipated is not constant in time. In order to test different cooling configurations of electronic components in the same working conditions, one measurement protocol was defined. It consisted of starting the personal computer and executing, in continuous loop, a simple computational program. Therefore, each electronic component dissipates a specific constant value of energy, and the steady state heat transfer with the air flow would be reached.

For the evaluation of the global (convective and radiation) heat transfer coefficient, we used a transient non-destructive method called pulsed photothermal radiometry method. When the steady-state heat exchange between the electronic components and the air flow is reached, a sudden supply of the luminous energy with fixed duration on the front face of the board was given by two halogen lamps (8). The analysis of the induced temperature elevation allows the calculation of the heat transfer coefficient between the air flow and the surface of each component. This is possible by the use of conduction model in the solid with a third-kind boundary condition at the interface. Two halogen lamps placed facing the main printed board, at the distance of $800 \mathrm{~mm}$, generate the finite duration excitations. The pulse duration, controlled by an electronic timer (9), can be fixed in the range 0.05 to 100 seconds with an accuracy of 0.01 second. On the back face of each lamp, a $400 \mathrm{~mm}$ length aluminium reflector permits to direct the energy uniformly on the surface of the main board. After the extinction of the lamps they are covered by a wood sheet, at the ambient temperature $T_{a m b}$, in order to eliminate the thermal radiation from the lamps (still hot) on the electronic board during its cooling by air.

\section{Temperature field}

In the original configuration of the main printed wiring board, heat is spread from the microprocessor through an aluminium heat sink. An axial fan allows the cooling of the heat sink by air in forced convection. Figure 2 represents the infrared photography of the original main board 20 minutes after starting the dismountable "personal computer". Axial fan, different diodes and capacitors, Integrated Power Electronic Modules (IPEM) which are the most heated components, are well distinguishable on this IR image. It was noticed that the temperature level was about $80^{\circ} \mathrm{C}$ on some components and around the microprocessor package. The former is not viewable because it is upon the heat sink, which is itself upon the axial fan.

Several IR images taken at different times during the functioning needed us to select 5 plane zones on the board where the temperature variation was high. These 
zones are indicated on the photography of the main board, taken after removing the axial fan with the heat sink from the microprocessor package (Figure 3).

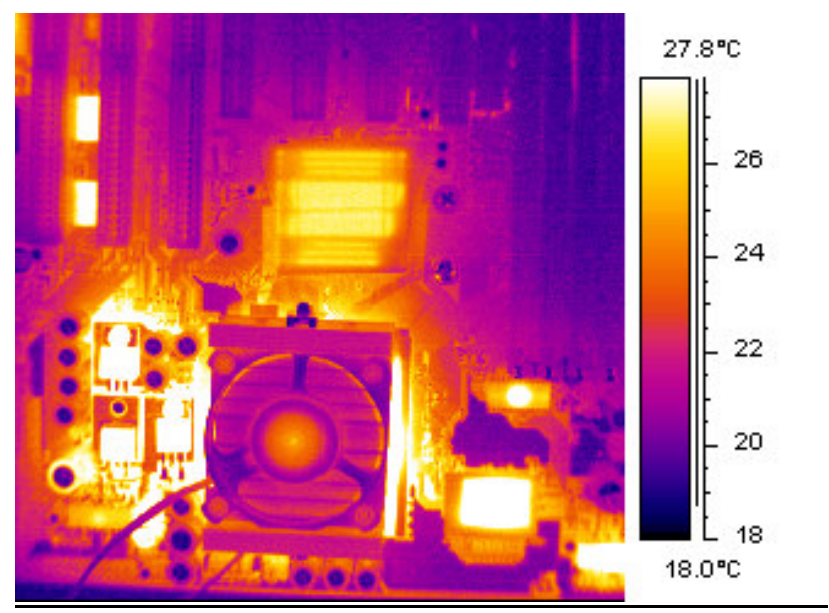

Fig. 2. Infrared photography of the main board in real situation with heat sink and axial fan on the microprocessor defined:

As mentioned above, an experimental procedure with three air velocity were $1 / \mathrm{t}=0$ second: starting working the main board with flow velocity $U_{\text {air }}^{1}=2.7 \mathrm{~m} \mathrm{~s}^{-1}$ $2 / \mathrm{t}=1800 \mathrm{~s}$, first change of the air velocity to $U_{\text {air }}^{2}=5.6 \mathrm{~m} \mathrm{~s}^{-1}$ $3 / \mathrm{t}=3000 \mathrm{~s}$, second change of the air velocity to $U_{\text {air }}^{3}=11 \mathrm{~m} \mathrm{~s}^{-1}$ 
http://dx.doi.org/10.21611/qirt.2006.085

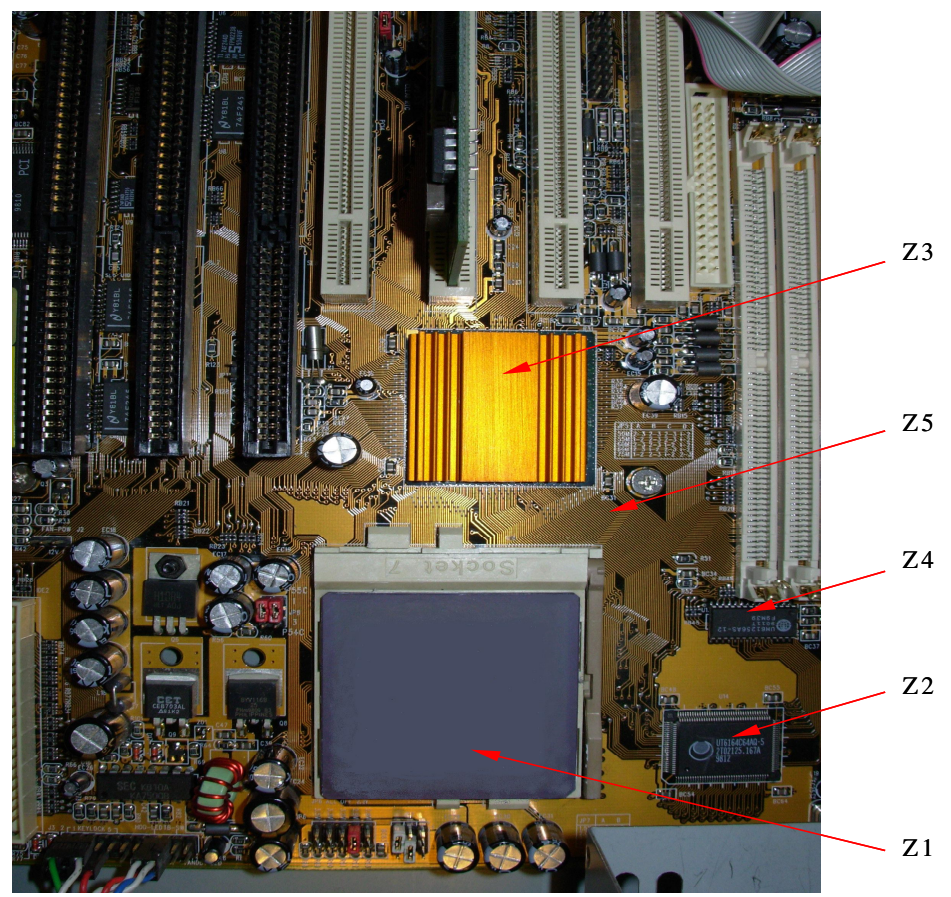

Fig. 3. Photography of the main board with the different zones

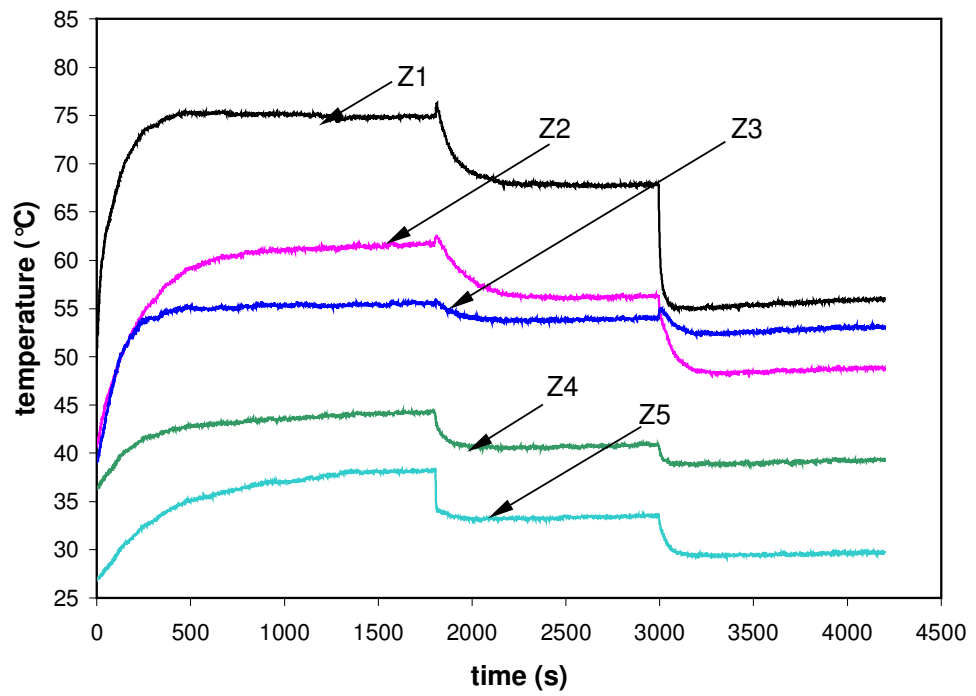

Fig. 4. Thermograms of the 5 zones on the main board 
The evolutions of the temperature at the 5 zones $Z 1, Z 2, \ldots Z 5$ are plotted versus the time on the figure 4 . It is noticed that, for each zone, the temperature growth in time and reach the first steady state corresponding to $U_{\text {air }}^{1}=2.7 \mathrm{~m} \mathrm{~s}^{-1}$. This state was reached faster for Z1 to Z4 than for Z5 which corresponds to a plane surface of the plastic frame. In addition, the temperature level diminished from Z1 to Z5. Indeed, the frame contains only very thin wiring connections between the components, so it is not heated and its thermal inertia is greater than those of the IPEM. In contrary, zones 1 to 4 correspond to IPEM with different level heating. When the flow velocity changed to $U_{\text {air }}^{2}=5.6 \mathrm{~m} \mathrm{~s}^{-1}$ the temperatures of all the zones decreased and reached the second steady state level. The third steady state is obtained after the change of the air flow generated by the double aspiration fan to $U_{\text {air }}^{3}=11 \mathrm{~ms}^{-1}$.

Infrared photography of the main board at a time $t=2500 \mathrm{~s}$ is given on the Figure 5. The temperature variation on the most heated element (microprocessor package) is well shown. This variation is due to the air flow generated by the fan from down side to the upper one of the main board. So the influence of the convective cooling on the variation of the temperature in the direction of the flow is evident.

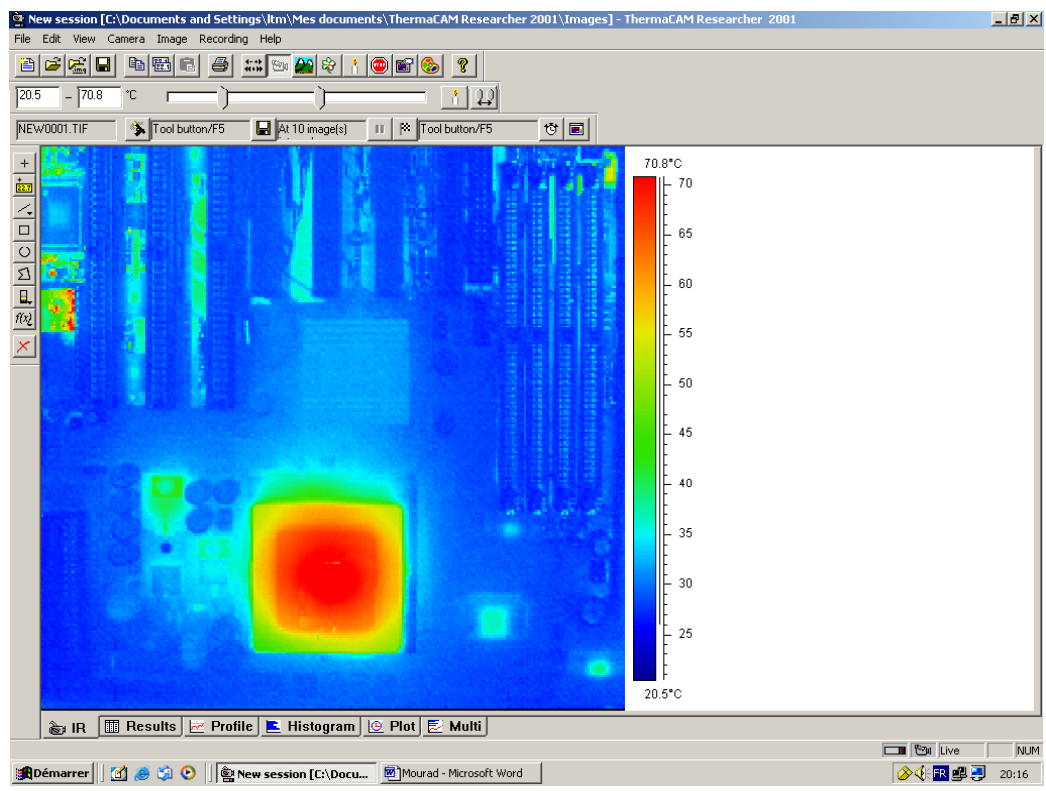

Fig. 5. Infrared photography of the main board without heat sink and axial fan on the microprocessor 


\section{http://dx.doi.org/10.21611/qirt.2006.085}

\section{Evaluation of the heat transfer coefficient}

In general, the evaluation of the convective heat transfer coefficient between the solid surface and the flow needs the installation of the flux and temperature sensors on the surface of the wall. The presence of these sensors generates, often, considerable disturbances on the heat transfer at the interfaces. This is why the use of a measurement technique without contact, as the photothermal radiometry method, has undeniable advantages. Being able to be employed in-situ, this technique consists in depositing energy on the front face of the wall, and recording the surface temperature by the infrared camera. This procedure is based on the flash method that was firstly introduced by Parker and al. [4] for the measurement of thermophysical properties of materials, such as the thermal diffusivity [5]. It was then extended to evaluate the heat transfer coefficients on different types of wall subjected to a convective heat exchange [6 - 10].

By examination of the transient temperature of the conduction model in the solid with third kind boundary condition, the heat transfer coefficient between the surface and the fluid may be evaluated if it is assumed to be constant in time. In other words, if one admits the assumption that the heat flux density remains linearly dependent of the surface temperature during the transient state. In previous papers $[9,10]$, we studied the influence of the pulse duration on the results of evaluation model with this assumption. The results given by the model were compared to those of a second model with heat transfer coefficient dependent of time, and with the measurement results by a multi-layered flux sensor in typical situations. Semi-infinite medium and isothermal thin wall had been studied, and several identification methods had been tested. It had been showed that the constant heat transfer coefficient assumption is debatable if the quantity of energy sent over the wall during the pulse affects considerably the boundary layer. The error induced may be of some tens per cent if the pulse duration is greater much than the time of the propagation of the thermal wave in the solid.

In actual study, the package of the microprocessor is thick about $E=4 \mathrm{~mm}$ and made of Epoxy moulded. From the database materiel web site their thermal properties are estimated to: $\lambda=0.4 \mathrm{~W} \mathrm{~m}^{-1} \mathrm{~K}^{1}$, for the conductivity and $a=1,610^{-7} \mathrm{~m}^{2}$ $\mathrm{s}^{-1}$ for the diffusivity. It is $54 \mathrm{~mm}$ large and $52 \mathrm{~mm}$ wide.

Due to the small thickness compared to the two others dimensions, the microprocessor can be assumed as a wall. The Biot number $B i=\frac{h E}{\lambda}$ varies from 0.1

to 1 if one considers that the heat transfer coefficient varies from $h=10$ to $100 \mathrm{~W} \mathrm{~m}^{-2}$ $K^{1}$ in air-flow. Hence, the one dimensional conduction model in the wall can be used. The propagation time of the heat wave in the wall thickness is about 10 seconds, so the assumption that $h$ remains constant in time is available from dirac excitation (very short pulse) to pulse duration less than 10 seconds.

The rise in temperature of the wall with finite thickness $(E)$ due to a brief thermal perturbation (Dirac) on its front face, verify the linear model $[10,11]$

$$
\begin{gathered}
\frac{\partial^{2} \theta}{\partial y^{2}}-\frac{1}{a} \frac{\partial \theta}{\partial t}=0 \\
y=0, \quad-\lambda \frac{\partial T}{\partial y}=h \theta(0, t)-W \delta(t) ; \quad \text { at } \quad t=0, \quad \theta(y, t)=0
\end{gathered}
$$


where $W$ is the energy density delivered by the halogen lamps, $h$ is the global heat transfer coefficient, $\delta(t)$ is the Dirac impulsion and $\theta(y, t)=T(y, t)-T_{\text {initial }}$ is the temperature elevation. By the Laplace Transform method, it was demonstrated that the temperature field in the packaging material is given by $[10,11]$

$$
\theta_{d}(x, t)=\frac{2 W}{\rho C E} \sum_{n=1}^{\infty} \frac{U_{n} \cos \left(U_{n}\left(1-\frac{x}{E}\right)\right)}{(1+B i) \sin U_{n}+U_{n} \cos U_{n}} \exp \left(-U_{n}^{2} \frac{a t}{E^{2}}\right)
$$

The expression of the temperature on the front face of the wall is:

$$
\theta_{d}(0, t)=\frac{2 W}{\rho C E} \sum_{n=1}^{\infty} \frac{U_{n} \cos \left(U_{n}\right)}{(1+B i) \sin U_{n}+U_{n} \cos U_{n}} \exp \left(-U_{n}^{2} \frac{a t}{E^{2}}\right)
$$

which can be arranged as:

$$
\theta_{d}(0, t)=\frac{2 W}{\rho C E} \sum_{n=1}^{\infty} \frac{U_{n}^{2}}{(1+B i) B i+U_{n}^{2}} \exp \left(-U_{n}^{2} \frac{a t}{E^{2}}\right)
$$

where $U_{n}$ is the nth-root of the transcendental equation $U_{n} \operatorname{tg}\left(U_{n}\right)=B i$.

The application of Duhamel's theorem by $[10,11]$ gives the response for a $\tau$ duration pulse with the heat flux density $\varphi$ :

$$
\begin{aligned}
& \text { if } t<\tau: \theta_{p}(0, t)=\frac{2 \varphi E}{\lambda} \sum_{n=1}^{\infty} \frac{U_{n}^{2}}{(1+B i) B i+U_{n}^{2}}\left[1-\exp \left(-U_{n}^{2} \frac{a t}{E^{2}}\right)\right] \\
& \text { if } t \geq \tau: \theta_{p}(0, t)=\frac{2 \varphi E}{\lambda} \sum_{n=1}^{\infty} \frac{U_{n}^{2}}{(1+B i) B i+U_{n}^{2}}\left[\exp \left(-U_{n}^{2} \frac{a(t-\tau)}{E^{2}}\right)-\exp \left(-U_{n}^{2} \frac{a t}{E^{2}}\right)\right]
\end{aligned}
$$

The identification of the heat transfer coefficient was based on the use of the partial temporal moment of the surface temperature. The analytical expression of the partial zero-order temporal moment of the surface temperature for a Dirac excitation is:

$$
m_{0}(t)=\int_{0}^{t} \theta_{d}(u) d u=\frac{2 W E}{\lambda} \sum_{n=1}^{\infty} \frac{1}{(1+B i) B i+U_{n}^{2}}\left[1-\exp \left(-U_{n}^{2} \frac{a t}{E^{2}}\right)\right]
$$

By the same way, the zero-order temporal moment of the surface temperature due to a $\tau$-duration pulse had been calculated. The temporal moment represents the total heat energy extracted by the air flow from the wall during the experiment time observation $t$. The calculation of $m_{0}(t)$ for several $B i$ number showed that when the pulse duration is less than the tenth of the observation time $t$, the temporal moment for Dirac excitation and $\tau$-duration excitation are quite different. In our experiment, a 3 second pulse was used. So, we identified the heat transfer coefficient by the use of 0 -order temporal moment of the thermogram on 30 seconds or more. Therefore, the temporal moment expression given in Eq (6) for Dirac excitation had been used for the identification. 


\section{http://dx.doi.org/10.21611/qirt.2006.085}

In practical way, for each experimental thermogram, given in figure 5 , the temporal moment was calculated by numerical integration. The heat transfer coefficient was identified by searching, by dichotomy, the value of $\mathrm{Bi}$ number which give the same theoretical temporal moment (Eq.4).

The identified global heat transfer coefficients are:

* $h^{1}=38 \mathrm{~W} \mathrm{~m}^{-2} \mathrm{~K}^{-1}$ for $U_{\text {air }}^{1}=2.7 \mathrm{~m} \mathrm{~s}^{-1}$

* $h^{2}=56 \mathrm{~W} \mathrm{~m}^{-2} K^{-1}$ for $U_{\text {air }}^{2}=5.6 \mathrm{~m} \mathrm{~s}^{-1}$

* $h^{3}=98 \mathrm{~W} \mathrm{~m}^{-2} \mathrm{~K}^{-1}$ for $U_{\text {air }}^{3}=11 \mathrm{~ms}^{-1}$

The total heat coefficient $h$ was assumed to be the sum of an $h_{c}$ coefficient (convection losses) and an $h_{r}$ coefficient (radiation losses). By using the linearization of the radiative heat transfer, the $h_{r}$ coefficient was calculated by:

$$
\left.\left.h_{r}=\varepsilon \sigma\left[T^{2}+T_{a m b}^{2}\right]\right] \mid T+T_{a m b}\right\rfloor
$$

where: $\varepsilon$ is the emissivity of the black paint (Nextel) deposed on the boarding card $(\varepsilon=0.95)$ and $\sigma$ is the Stephan-Boltzmann constant.

Application of Eq. (7) showed that the $h_{r}$ coefficient is practically constant in for the three thermograms and has an average value: $h_{r}=7 \mathrm{~W} \mathrm{~m}^{-2} \mathrm{~K}^{-1}$. Thus, the convective heat transfer coefficient could be obtained by: $h_{c}=h-h_{r}$. The $h_{c}$ values calculated are:

* $h_{c}^{1}=30 \mathrm{~W} \mathrm{~m}^{-2} \mathrm{~K}^{-1}$ for $U_{\text {air }}^{1}=2.7 \mathrm{~m} \mathrm{~s}^{-1}$

* $h_{c}^{2}=49 \mathrm{~W} \mathrm{~m}^{-2} \mathrm{~K}^{-1}$ for $U_{\text {air }}^{2}=5.6 \mathrm{~m} \mathrm{~s}^{-1}$

* $h_{c}^{3}=91 \mathrm{~W} \mathrm{~m}^{-2} \mathrm{~K}^{-1}$ for $U_{\text {air }}^{3}=11 \mathrm{~m} \mathrm{~s}^{-1}$

The results are of the same order of the known values of the heat transfer coefficient with air-forced convection on flat plate. They show the variation of the efficiency of the cooling by increasing the air velocity. A comparison with the results available in the literature is impossible here since the former were established under conditions different from ours and for well defined flows. Which is not the case here, where we seek to evaluate the heat transfer coefficient between the finite thickness wall and the air flow of unknown nature (laminar, turbulent, flow parallel to solid, with singularity...).

\section{Conclusion}

Cartographies of the thermal field during the real functioning conditions of a main printed wiring board of a PC are given. We have shown the possibility to evaluate the heat transfer coefficient from one electronic power module by the pulsed photothermal method. The results given constitute the reference data for the study the heat convective improvement in the thermal management of the air-cooling systems used in electronic.

\section{References}

[1] S. Kakaç, L.L. Vasiliev; Y. Bayazitoglu and Y. Yener, Microscale Heat Transfer, NATO Sciences Series, Springer, (2005) 
[2] A.S. Huzayyin, S.A. Nada, M.A. Rady and A. Faris, Cooling an array of multiple heat sources by a row of slot air jet, Int. J. Heat Mass Transfer 49 (2006) 25972609

[3] M.M. Mohammed, Air cooling characteristics of uniform square modules array for electronic device heat sink, J. App. Thermal Engineering 26 (2006) 486-493

[4] W.J. Parker, R.J. Jenkins, C.P. Butler and G.L. Abbott, Flash method in determining thermal diffusivity, heat capacity and thermal conductivity", J. Appl. Phys. 32 (1961) 1679-1684

[5] A. Degiovanni, Identification de la diffusivité thermique par l'utilisation des moments temporels partiels, High Temperatures-High Pressures 17 (1985) 683-689.

[6] D.L. Balageas, D. M. Boscher, A.A. Deom, J. Fournier et G. Gardette, Application de la thermographie infrarouge passive et stimulée aà la mesure des flux thermiques en soufflerie, La Recherche Aeospatiale 4 (1991) 51-72.

[7] M. Remy, A. Degiovanni et D. Maillet, Mesure de coefficient d'échange pour des écoulements à faible vitesse, Revue Génénale Thermique (Int. J. Thermal Sciences) 397 (1995) 28-42

[8] D.J. Crowther and J. Padet, Measurement of the local convection coefficient by pulsed photothermal radiometry, Int. J. Heat Mass Transfer, 34 (1991) 30753081

[9] M. Rebay, M. Lachi et J. Padet, Mesure de coefficients de convection par méthode impulsionnelle - Influence de la perturbation de la couche limite, Int. J. Thermal Sciences, 41 (2002) 1161-1175

[10] M. Rebay, M. Lachi and J.F. Henry, Influence of the plate roughness on the convective heat transfer coefficient, Visualization, Imaging and Data Analysis In Convective Heat and Mass Transfer (2002)

[11] S.Kakac and Y.Yener, Heat Conduction, third edition, Taylor and Francis, (1993)

[12] F.P. Incropera and D.P.DeWitt, Introduction to heat transfer, third edition, John Wiley, (1996)

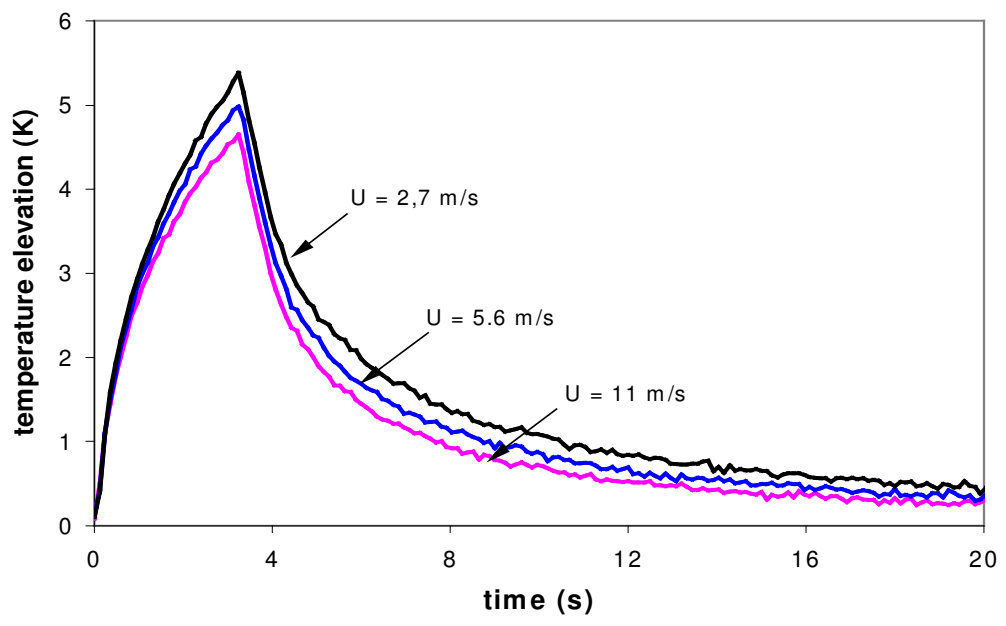

Fig. 5. Responses to 3 seconds pulsed excitation with 3 flow velocities 